\title{
E-cadherin and ZEB2 modulate trophoblast cell differentiation during placental development in pigs
}

\author{
Linjun Hong${ }^{1}$, Kun Han¹, Kejia Wu¹, Ruize Liu ${ }^{1}$, Ji Huang ${ }^{1}$, Joan K Lunney², Shuhong Zhao ${ }^{1}$ and \\ Mei $\mathrm{Yu}^{1}$ \\ ${ }^{1}$ Key Lab of Agricultural Animal Genetics, Breeding and Reproduction of Ministry of Education, The Cooperative \\ Innovation Center for Sustainable Pig Production, Huazhong Agricultural University, Wuhan, China and ${ }^{2}$ U.S. \\ Department of Agriculture, Animal Parasitic Diseases Laboratory, Beltsville Agricultural Research Center, Agricultural \\ Research Service, Beltsville, Maryland, USA \\ Correspondence should be addressed to M Yu; Email: yumei@mail.hzau.edu.cn
}

\begin{abstract}
It is one of the important events that trophoblast cells within the placental folds differentiate into two types that differ in cell shape during placental development in pigs. This study showed that all the trophoblast cells were of similar shape between Yorkshire and Chinese Meishan pigs on day 26 of gestation; thereafter, the trophoblast cells located at the top of the placental folds became high columnar, while those cells at the base of the placental folds were cuboidal on day $\mathbf{5 0}$ of gestation. Additionally, on day 95 of gestation, all the trophoblast cells in Meishan pigs became cuboidal, but the trophoblast cells located at the top of the placental folds in Yorkshire pigs still remained columnar. The membranous E-cadherin and $\beta$-catenin were strongly co-expressed by the high columnar trophoblast cells but very weakly expressed by those cuboidal cells. Consistently, the expression pattern of ZEB2, the E-cadherin repressor, was inversely correlated with that of E-cadherin in the two types of trophoblast cells in the two breeds. Furthermore, electrophoretic mobility shift assays demonstrated the binding of ZEB2 to the E-cadherin promoter in nuclear extracts from porcine placental tissue. These findings suggest a ZEB2-dependent mechanism of trophoblast cell differentiation during placental development in pigs.

Reproduction (2017) 154 765-775
\end{abstract}

\section{Introduction}

In mammals, the placenta is of dominant importance for the intrauterine development and growth of the fetus throughout the whole pregnancy. The establishment of a firm contact between conceptus and mother can enable the efficient exchange of substances between the maternal and fetal blood vessels. Placentation begins with implantation which includes specialized cell migration and adhesion, leading to attachment of conceptus trophoblast to the maternal uterine luminal epithelium (Song et al. 2010). In order to increase placental efficiency, placental and endometrial tissues undergo remodeling to reduce the distance and increase the maternal-fetal interacting surface area as gestation advances. This occurs in all mammals regardless of the types of placentas (Enders \& Carter 2004, Song et al. 2010). In pigs, placental dysfunction can result in fetal loss during gestation and depression in piglet birth weight which is a primary factor associated with pre-weaning mortality (Tuchscherer et al. 2000, Mesa et al. 2006, Vallet et al. 2014). Thus, it is necessary to understand how the placenta functions and what factors influence placental efficiency.

Pigs have a diffuse, epitheliochorial type of placenta (Leiser et al. 1998). The trophoblast epithelial layer starts to attach to the maternal endometrial epithelial layer (uterine luminal epithelium) to form the epithelial bilayer around days 15-20 of gestation. The non-invasive epitheliochorial placenta is established around days 26-30 of gestation (Dantzer 1985, Leiser et al. 1998). Thereafter, the pig placenta undergoes remodeling to maximize the placental efficiency as gestation advances by: (1) increasing the maternal-fetal exchange area through the development of placental folds (Vallet \& Freking 2007, Miles et al. 2009); (2) reducing the distance between maternal and fetal capillaries (Friess et al. 1980, Leiser \& Dantzer 1988, Vallet et al. 2013) and (3) enhancing the nutrient-specific transport (Raub et al. 1985, Kim \& Vallet 2007, Hong et al. 2016). In addition, previous research revealed that during gestation, the trophoblast cells within the placental folds differentiate into two types of cells which differ in cell shape and are located at the top and base of the placental folds, respectively. Therefore, changes in the morphological 
features of the trophoblast cells have crucial influence on placental development in pigs. However, the mechanisms involved in changes of the trophoblast cell shape during placental development in pigs remain to be fully elucidated (Miles et al. 2009, Vallet et al. 2010, Hong et al. 2014, Vallet et al. 2014).

In epithelial tissues, cell-cell adhesion is essential for the maintenance of the epithelial cell morphology and function. E-cadherin acts as a master regulator of epithelial cell adhesion (Hay 1995, Daikoku et al. 2011). E-cadherin is a single-span transmembrane, $\mathrm{Ca}^{2+}$ dependent cell-adhesion molecule consisting of five extracellular cadherin-binding domains and a conserved intracellular domain that associates with catenins (Takeichi 1995, Reardon et al. 2012). E-cadherinmediated cell-cell adhesion is accomplished through homophilic binding between E-cadherins on the surface of adjacent cells, and the adhesion is stabilized by association with $\beta$-catenin which links the cytoplasmic terminal tail of E-cadherin to the actin cytoskeleton (Auersperg et al. 1999, Leckband \& Sivasankar 2012). Studies have demonstrated that most tumor cells which lose cell-cell adhesion and/or undergo the epithelialmesenchymal transition (EMT) are associated with the decrease or absence of E-cadherin expression (Thiery 2002, Kokkinos et al. 2010, Zhang et al. 2016). Evidence suggests that E-cadherin plays important roles in regulation, differentiation and the EMT of human placental trophoblast cells (Zhou et al. 1997, Kokkinos et al. 2010, Du et al. 2016). ZEB1 (zinc finger E-box binding homeobox 1) and ZEB2 (zinc finger E-box binding homeobox 2) are members of the zinc finger homeobox family. These two transcript factors suppress the E-cadherin expression by binding to E2-boxes in the E-cadherin promoter in several types of epithelial cells (Comijn et al. 2001, Eger et al. 2005, Kokkinos et al. 2010). Although E-cadherin and its regulators are widely considered determinants of epithelial cell differentiation, there is no information concerning their role in trophoblast cell differentiation during pig placental development.

Chinese Meishan pigs are reproductively very prolific (Ford \& Youngs 1992, Youngs et al. 1993). Our previous study showed that the placental folds during late gestation in Meishan pigs are more complex than those in the less prolific Yorkshire pigs. The increased complexity of placental folds was suggested as a mechanism that enables Meishan pigs to have higher placental efficiency when compared to Yorkshire pigs (Hong et al. 2014). However, there are little data about morphology and function of trophoblast cells during the placental development in the two breeds. Thus, in the present study, we evaluated: (1) morphological changes of the trophoblast cells in Yorkshire and Meishan pigs during different stages of placental development (day 26 of gestation, initiation stage; day 50 of gestation, establishment stage and day 95 of gestation, late development stage; gestation length for pigs is 114 days) and (2) the role of E-cadherin and its regulator ZEB2 in morphological changes of the trophoblast cells in pigs.

\section{Materials and methods}

\section{Animals and tissue collection}

All procedures for the collection of samples were approved by the Ethics Committee of Huazhong Agricultural University, P.R. China. Gilts used for this experiment were described by Hong et al. (2013). Briefly, Chinese Meishan and Yorkshire gilts were checked for estrus twice daily and mated naturally at their second estrous cycle after puberty to boars of their own breed at the onset of estrus (day 0 ) and again $12 \mathrm{~h}$ later. The gilts were killed on days 26, 50 and 95 of gestation ( $n=3-4$ gilts/breed/day of gestation). The uteri were quickly removed and transported in an icebox to the laboratory where they were opened longitudinally along the anti-mesometrial side. For each gilt, rectangular sections of the fetal/placental units (including myometrium, endometrium, and placenta) located closed to the umbilical cord were collected and fixed immediately in fresh $4 \%$ paraformaldehyde in phosphatebuffered saline (PBS; $\mathrm{pH}: 7.2$ ) for $24 \mathrm{~h}$ followed by paraffin embedding (FFPE) for histological examination and immunohistochemistry. The placental tissue for each fetus was collected, snap-frozen in liquid nitrogen and stored at $-80^{\circ} \mathrm{C}$ until protein was extracted.

\section{Histomorphometry}

In order to measure and analyze cell morphometric parameters, H\&E staining was performed as described by Hong et al. (2013). Briefly, the paraffin-embedded tissues were sectioned $(4-\mu \mathrm{m}$ thick), placed on SuperfrostPlus slides (Thermo Scientific), processed through a graded series of xylene and ethanol, stained with hematoxylin and eosin and placed under a cover slip. A Nikon 80i light microscope fitted with a Nikon (DS-Fi1) digital camera and the NIS-Elements 3.2 software (Nikon) was used for cell morphometric measurements. For each gilt, 3 uterine/placental units were studied as individual samples.

\section{PAS staining}

Periodic acid-Schiff (PAS) stain was performed as the protocol (Wuhan Goodbio Technology Co., Ltd., Wuhan, China). Briefly, sections (4- $\mu \mathrm{m}$ thick) were deparaffinized with xylene and rehydrated in an alcohol gradient, 100\% ethanol, 95\% ethanol, $90 \%$ ethanol, $80 \%$ ethanol, $70 \%$ ethanol and distilled water. The sections were incubated with periodic acid solution at room temperature for $10 \mathrm{~min}$. After rinsing with distilled water, sections were then incubated with Schiff's reagent at room temperature from light for $10 \mathrm{~min}$. Afterward, sections were counterstained with hematoxylin and mounted. All sections were stained under the same conditions. Images of the PAS staining were taken by an Olympus microscope BX-53 with a digital camera DP26 (Olympus). Photographic images were assembled using Adobe Photoshop CS6 (Adobe Systems Inc.). 


\section{Immunohistochemistry}

Immunohistochemical analysis was performed as previously described (Hong et al. 2016). Sections (4- $\mu \mathrm{m}$ thick) were deparaffinized with xylene and rehydrated in an alcohol gradient $(100 \%$ ethanol, 95\% ethanol, 90\% ethanol, $80 \%$ ethanol, $70 \%$ ethanol and distilled water), and then treated with $3 \%$ hydrogen peroxide $\left(\mathrm{H}_{2} \mathrm{O}_{2}\right)$ to block endogenous peroxidase for $15 \mathrm{~min}$ at room temperature. After rinsing with distilled water, sections were submitted to heat-induced epitope retrieval by treatment in $0.01 \mathrm{M}$ sodium citrate buffer (pH 6.0) in a microwave oven at $750 \mathrm{~W}$ for $15 \mathrm{~min}$ (thrice for 5 min each) (Krenacs et al. 2010). Afterward, sections were cooled for $30 \mathrm{~min}$ at room temperature and rinsed thrice in PBS, 5 min each. The sections were blocked with $5 \%$ bovine serum albumin in PBS for 30 min in a humid chamber at room temperature. The sections were incubated with antibodies at $4{ }^{\circ} \mathrm{C}$ overnight, rinsed and then incubated with secondary antibody for $30 \mathrm{~min}$. Specific information on antibodies was noted in Supplementary Table 1 (see section on supplementary data given at the end of this article). Following immunostaining, sections were counterstained with hematoxylin and mounted. For each sample, a negative control (NC) was performed by replacing the primary antibody with corresponding nonspecific immunoglobulin G (IgG). All sections were stained by immunohistochemistry for each antibody under the same conditions. Images were taken by an Olympus microscope BX-53 with a digital camera DP26 (Olympus). Photographic images were assembled using Adobe Photoshop CS6 (Adobe Systems Inc.). In order to quantify the immunohistochemical staining intensity, mean integrated optical density (IOD) was calculated using ImagePro Plus 6.0 software (Media Cybernetics, Silver Spring, USA) as described previously (Hong et al. 2014). For each gilt, 3 uterine/placental units were studied as individual samples.

\section{Co-immunoprecipitation (Co-IP)}

Porcine placental tissues obtained on day 26 of gestation were lysed in cold RIPA lysis buffer (Beyotime Biotechnology, Shanghai, China) with protease inhibitors (1 mM PMSF, $5 \mathrm{mM}$ $\mathrm{NaVO}_{4}, 10 \mu \mathrm{g} / \mathrm{mL}$ leupeptin and $10 \mu \mathrm{g} / \mathrm{mL}$ aprotinin) and then incubated on ice for $20 \mathrm{~min}$. The lysates were centrifuged $\left(18000 \mathrm{~g}, 30 \mathrm{~min}, 4^{\circ} \mathrm{C}\right)$ and the supernatant was collected. AntiE-cadherin (Abcam, Rabbit monoclonal, ab40772) and normal rabbit IgG were crosslinked by Dynabeads Protein A (Thermo 10001D) at $4^{\circ} \mathrm{C}$ overnight. The supernatant was incubated with antibody-conjugated Dynabeads and IgG-conjugated Dynabeads, and rotated for $12 \mathrm{~h}$ at $4{ }^{\circ} \mathrm{C}$. The protein-antibody complexes were collected by magnet and washed for 6 times with IP buffer. After being heated at $70^{\circ} \mathrm{C}$ for $10 \mathrm{~min}$, the immunoprecipitated complexes were released for the western blot analysis.

\section{Western blot}

Proteins were mixed equally with SDS loading buffer and resolved on SDS-PAGE gel. Proteins on the gel were then transferred onto PVDF membranes (Roche). After blocking with $5 \%$ milk protein in TBST for $3 \mathrm{~h}$, the membranes were incubated with E-cadherin (mouse monoclonal, 60335-1-Ig, Proteintech) and $\beta$-catenin (mouse monoclonal, 66379-1-lg, Proteintech) antibodies at $4^{\circ} \mathrm{C}$ overnight. Then, the blots were washed thrice with TBST, and incubated for $2 \mathrm{~h}$ with HRP-conjugated secondary antibodies. Immunolabeling was detected by ECL (Pierce) after washing with TBST.

\section{Electrophoretic mobility shift assays (EMSA)}

It has been demonstrated that ZEB2 downregulates the E-cadherin expression by binding to E2-boxes which were the conserved regulatory elements in the E-cadherin promoter region (Comijn et al. 2001, Van Aken et al. 2001). Thus, we designed the oligonucleotides containing E2-box element based on porcine E-cadherin sequence (accession No: NC_010448). The sequences corresponding to these oligonucleotides are shown in Fig. 7. The oligonucleotides were synthesized commercially (Sangon, Shanghai). By using NE-PER nuclear and cytoplasmic extraction reagent kit (ThermoFisher), nuclear extracts were prepared from placental tissues of Meishan pigs obtained on day 95 of gestation. By using LightShift chemiluminescent EMSA kit (ThermoFisher), EMSA were performed with $3 \mu \mathrm{g}$ placental nuclear extracts, $20 \mathrm{fmol}$ biotin-labeled double-stranded oligonucleotides, $10 \times$ binding buffer and $1 \mu \mathrm{g}$ poly $(\mathrm{dl}-\mathrm{dC})$. For supershift assays, $1 \mu \mathrm{g}$ ZEB2 antibody (Abcam) was added to each reaction. The protein-DNA complexes were resolved by electrophoresis in $5.5 \%$ polyacrylamide gels, and then transferred to a nylon membrane. The dried nylon was scanned with GE ImageQuant LAS4000 mini (GE Healthcare).

\section{Statistical analysis}

Immunohistochemical staining intensity data were analyzed using PROC MIXED in SAS (Version 8.1; SAS Institute, Inc., Cary, NC, USA). The model used included the fixed effects of gestational day (gestational days 26, 50 and 95), breed (Yorkshire and Meishan) and breed $\times$ gestational day interaction. Gilt within breed $\times$ gestational day was included as a random effect. A significance level of $P<0.05$ was considered significant.

\section{Results}

\section{Histological examination of the placental trophoblast cells in pigs}

The height and width of the trophoblast cells at different developmental stages of pig placenta were measured and the data are listed in Table 1. On day 26 of gestation, the trophoblast cells were similar in shape (Yorkshire: $17.93 \times 8.38 \mu \mathrm{m}$; Meishan: $16.40 \times 8.26 \mu \mathrm{m}$ ) (Fig. 1 and Table 1). On day 50 of gestation, the trophoblast cells at different locations of the placental folds showed morphological differences. At the base of the placental folds (oriented with fetal side of the placenta as 'up'), the trophoblast cells remained cuboidal in shape (Yorkshire: $12.75 \times 6.19 \mu \mathrm{m}$; Meishan: $12.82 \times 6.29 \mu \mathrm{m})$. However, at the top of the placental folds, the trophoblast 
Table 1 Characteristics of Meishan and Yorkshire trophoblast cells during gestation.

\begin{tabular}{|c|c|c|c|c|c|c|c|c|}
\hline \multirow{3}{*}{$\begin{array}{l}\text { Days of } \\
\text { gestation }\end{array}$} & \multicolumn{4}{|c|}{ Height of the trophoblast $(\mu \mathrm{m}$, mean \pm S.D. $)$} & \multicolumn{4}{|c|}{ Wide of the trophoblast $(\mu \mathrm{m}$, mean \pm S.D. $)$} \\
\hline & \multicolumn{2}{|c|}{ Yorkshire } & \multicolumn{2}{|c|}{ Meishan } & \multicolumn{2}{|c|}{ Yorkshire } & \multicolumn{2}{|c|}{ Meishan } \\
\hline & Top of the fold & Base of the fold & Top of the fold & Base of the fold & Top of the fold & Base of the fold & Top of the fold & Base of the fold \\
\hline 26 & $17.93 \pm 1.31$ & 1 & $16.40 \pm 1.05$ & 1 & $8.38 \pm 0.43$ & / & $8.26 \pm 0.69$ & l \\
\hline 50 & $42.08 \pm 3.64$ & $12.75 \pm 1.34$ & $40.37 \pm 3.47$ & $12.82 \pm 1.36$ & $4.62 \pm 0.28$ & $6.19 \pm 0.53$ & $4.47 \pm 0.48$ & $6.29 \pm 0.70$ \\
\hline 95 & $22.54 \pm 0.89$ & $9.56 \pm 1.10$ & $18.25 \pm 0.85$ & $15.98 \pm 0.98$ & $4.79 \pm 0.23$ & $9.38 \pm 0.50$ & $12.21 \pm 0.91$ & $13.88 \pm 1.16$ \\
\hline
\end{tabular}

/, absent.

cells became high columnar in shape (Yorkshire, $42.08 \times 4.62 \mu \mathrm{m}$; Meishan, $40.37 \times 4.47 \mu \mathrm{m})$ (Fig. 1 and Table 1). Thus, the trophoblast cells changed their size and shape from day 26 to 50 of gestation but there were no obvious differences in cell size and shape between the two pig breeds.

On day 95 of gestation, the trophoblast cellular morphology was different between the two breeds. In Yorkshire pigs, the trophoblast cells located at the top of the placental folds still remained columnar $(22.54 \times 4.79 \mu \mathrm{m})$ although the cell height was reduced. In contrast, the trophoblast cells located at the base of

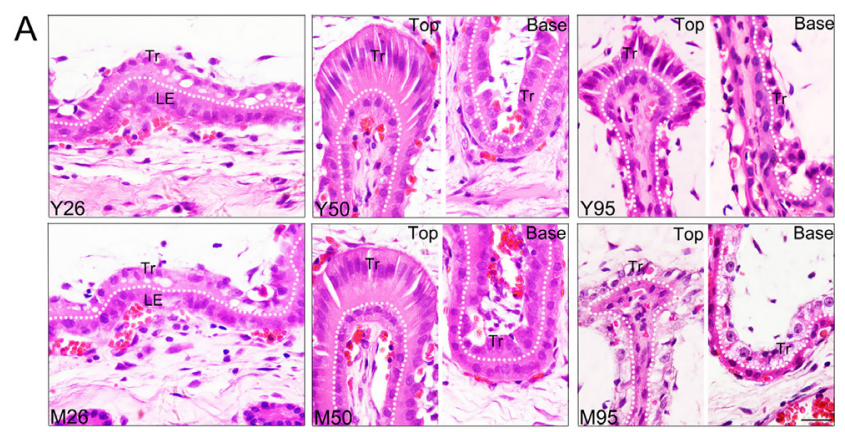

B

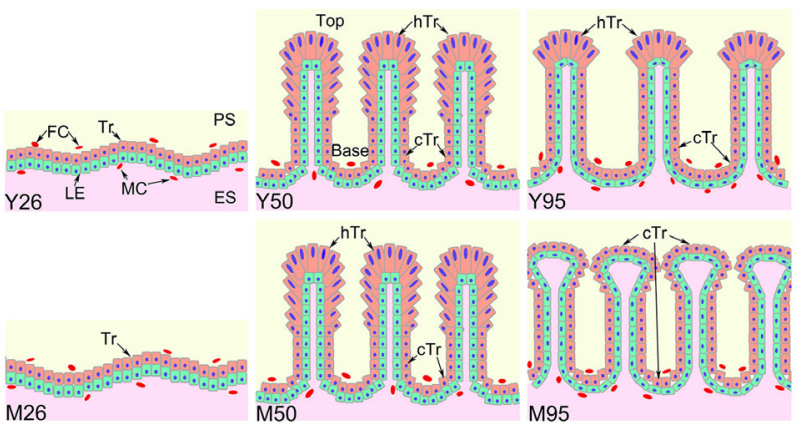

Figure 1 Morphologic changes of trophoblast cells during placental development in Yorkshire $(\mathrm{Y})$ and Meishan $(\mathrm{M})$ pigs. (A) HE staining in representative uterine/placental sections obtained from days 26, 50 and 95 of gestation in Yorkshire (Y) and Meishan (M) pigs. Tr, trophoblast; LE, endometrial luminal epithelium; Top, top of placental folds; Base, base of placental folds. Scale bars $=20 \mu \mathrm{m}$. (B) Schematic drawing of the trophoblast (Tr) differentiation as gestation advances in pig. Tr, trophoblast; hTr, high columnar trophoblast cells; cTr, cuboidal trophoblast cells; LE, endometrial luminal epithelium; FC, fetal capillaries; MC, maternal capillaries; PS, placental stroma; ES, endometrial stroma; Top, top of placental folds; Base, base of placental folds. the folds became cuboidal in shape $(9.56 \times 9.38 \mu \mathrm{m})$ (Fig. 1 and Table 1). However, in Meishan pigs, the trophoblast cells located both at the top and the base of the placental folds were cuboidal in shape (Fig. 1 and Table 1). In addition, the cuboidal trophoblast cells located at the base of the placental folds in Meishan pigs were larger in size, when compared with those in Yorkshire pigs (Yorkshire: $9.56 \times 9.38 \mu \mathrm{m}$; Meishan: $15.98 \times 13.88 \mu \mathrm{m})$ (Fig. 1 and Table 1). Taken together, these findings indicate that the shape and size of trophoblast cells changed during placental development in pigs.

\section{The expression patterns of $E$-cadherin and $\beta$-catenin in trophoblast cells during placental development in pigs}

On days 26 and 50 of gestation, the expression pattern of E-cadherin in trophoblast cells was similar in Yorkshire and Meishan pigs (Fig. 2A and C). On day 26, E-cadherin was expressed uniformly in trophoblast cells and the positive signals were localized at the cell membranes. On day 50, positive staining signal of E-cadherin was detected in the columnar trophoblast cells located at the top of the placental folds but rare or undetectable in cuboidal trophoblast cells at the base and side of the placental folds. On day 95 of gestation, the expression pattern of E-cadherin was different between the two breeds (Fig. 2A and C). In Yorkshire pigs, the columnar trophoblast cells located at the top of the placental folds still had the intense staining of E-cadherin, whereas the cuboidal trophoblast cells located at the base and side of the placental folds showed very weak staining. However, in Meishan pigs on day 95 of gestation, all trophoblast cells which were cuboidal in shape did not express E-cadherin. $\beta$-Catenin can link E-cadherin in the adherent junction. We found that the positive staining signal for $\beta$-catenin was also localized at the cell membranes. In addition, the expression pattern of $\beta$-catenin was exactly the same as that of E-cadherin on days 26, 50 and 95 of gestation in the two breeds (Fig. 2B and D). Thus, E-cadherin and $\beta$-catenin were co-expressed in trophoblast cells and changes in their expression patterns were associated with changes in trophoblast cell shape during placental development in pigs.

Subsequently, we investigated whether E-cadherin interacts with $\beta$-catenin in trophoblast cells in pigs. 
A

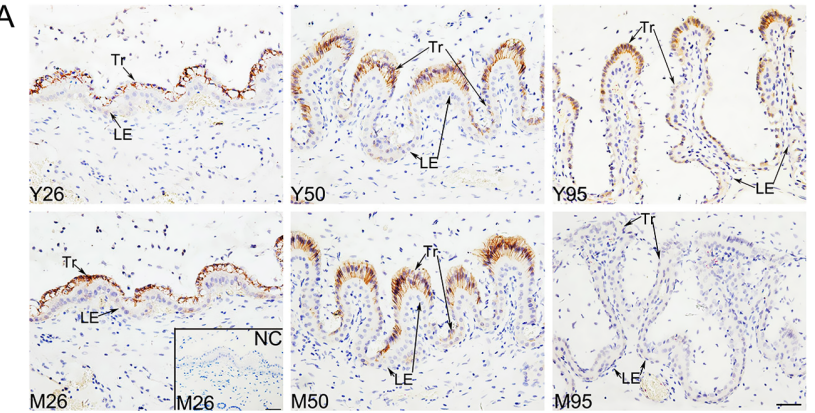

B

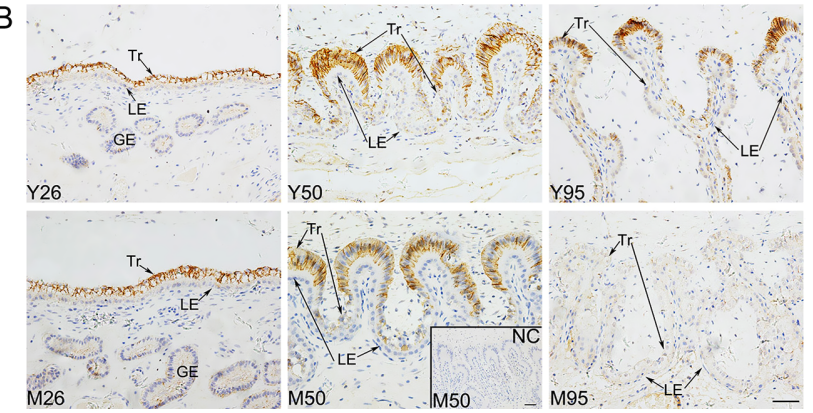

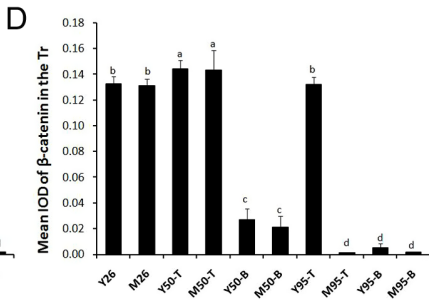

Figure 2 Immunohistochemical analysis of $\mathrm{E}$-cadherin and $\beta$-catenin at the maternal-fetal interface in Yorkshire $(\mathrm{Y})$ and Meishan $(\mathrm{M})$ pigs. (A) E-cadherin expression pattern at the maternal-fetal interface during placental development. On day 26 of gestation, E-cadherin stainings were observed in all trophoblasts $(\mathrm{Tr})$ in $\mathrm{Y}$ and $\mathrm{M}$ pigs. On day 50 of gestation, positive signals were mostly observed in Tr cells located at the top of the placental folds in $\mathrm{Y}$ and M pigs. On day 95 of gestation, positive signals were still observed in Tr cells located at the top of the placental folds in $\mathrm{Y}$, while in $\mathrm{M}$, the positive staining was not detected. (B) $\beta$-Catenin expression pattern at the maternalfetal interface during placental development. The expression pattern of $\beta$-catenin was exactly same with the expression pattern of E-cadherin on days 26, 50 and 95 of gestation in $\mathrm{Y}$ and $\mathrm{M}$ pigs. $\mathrm{Tr}$, trophoblast; LE, endometrial luminal epithelium. Insert: NC, negative control; scale bars $=40 \mu \mathrm{m}$. (C) Quantitative analysis of E-cadherin by measuring the average integrated optical density (IOD) in trophoblast cells during placental development. (D) Quantitative analysis of $\beta$-catenin by measuring the average integrated optical density (IOD) in trophoblast cells during placental development. Means of immunoreaction intensity with different superscript letters differ significantly at $P<0.05$. The data were shown as the mean \pm S.D. T, top of placental folds; B, bottom of placental folds.

Due to our findings in this study that E-cadherin and $\beta$-catenin were expressed exclusively by trophoblast cells in porcine placentas on day 26 of gestation (Fig. 2), co-IP assays were performed using lysate from placental tissues obtained on day 26 of gestation.

M26

\begin{tabular}{l|l}
\hline Lysate IP:IgG IP: E-cadherin \\
\\
IB:E-cadherin \\
\\
IB: $\beta$-catenin
\end{tabular}

Figure $3 \mathrm{Co}-\mathrm{IP}$ analysis of E-cadherin/ $\beta$-catenin interactions in porcine placental tissue. IP, immunoprecipitation; IB, immunoblotting M26, placentas of Meishan pigs from day 26 of gestation.

The results indicate that the interaction between E-cadherin and $\beta$-catenin in porcine trophoblast cells was detected (Fig. 3).

\section{Trophoblast cell PAS staining}

PAS staining was performed for detecting the presence of the carbohydrate macromolecules (glycogen, glycoprotein and proteoglycans) in the trophoblast cells. On day 26 of gestation, cytoplasmic granules that were strongly stained with PAS accumulated at the basal pole of the trophoblast cells in both Yorkshire and Meishan pigs (Fig. 4). On day 50 of gestation, positive PAS staining was seen in all the trophoblast cells in the two breeds, but the staining density was much stronger in
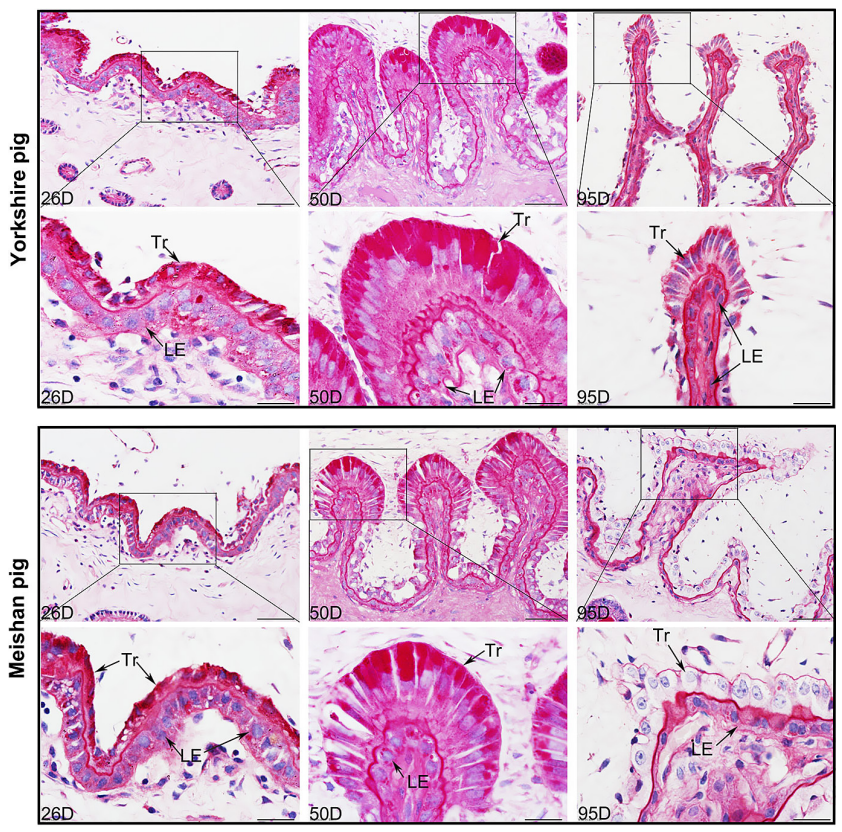

Figure 4 PAS staining at the maternal-fetal interface on days 26, 50, and 95 of gestation in Yorkshire and Meishan pigs. Positive signals (red granules) were mostly observed in the high columnar trophoblast cells. Tr, trophoblast; LE, endometrial luminal epithelium. Scale bars $=40 \mu \mathrm{m}$ or $20 \mu \mathrm{m}$ (amplification). 
the trophoblast cells located at the top of the folds than in those at the base. On day 95 of gestation, the PAS staining pattern in Yorkshire pigs was similar with that on day 50 of gestation. In contrast, in Meishan pigs, very few PAS positive granules were found in the trophoblast cells located either at the top or at base of the folds (Fig. 4). These findings suggest that the secretion of carbohydrate macromolecules may be enhanced in the trophoblast cells which were columnar and E-cadherin-positive.

\section{Differential expression patterns of transcription factors ZEB1 and ZEB2 in trophoblast cells during placental development in pigs}

Although positive staining for ZEB1 was observed in a few stromal cells, no positive staining was seen in any trophoblast cells from either breed during the three stages of gestation (Fig. 5A). However, positive signals for ZEB2 were detected in the nuclei of E-cadherin-negative trophoblast cells (Fig. 5B). On day 26 of gestation, few positive ZEB2 signals were detected in trophoblast cells. On day 50, a few positive ZEB2 signals were detected in the cuboidal trophoblast cells which were located at the base and sides, but not at the top, of the placental folds in the two breeds. On day 95 of gestation, the expression pattern of ZEB2 was different between the two breeds (Fig. 5B and C). In Yorkshire pigs, only the cuboidal trophoblast cells located at the base and sides of the placental folds had positive staining for ZEB2. However, in Meishan pigs, all the trophoblast cells which were cuboidal in shape expressed ZEB2 on day 95 of gestation. These results indicate that ZEB2 was strongly expressed by cuboidal trophoblast cells and changes in its expression pattern were associated with changes in trophoblast cell shape during placental development in pigs.

\section{Relationship between expression patterns of ZEB2 and E-cadherin in porcine trophoblast cells}

We performed further investigation to demonstrate the relationship between expression patterns of ZEB2 and $\mathrm{E}$-cadherin in trophoblast cells during gestation. Because primary antibodies for ZEB2 and E-cadherin raised in different species were not available commercially, co-immunostaining procedure cannot be carried out in this study. Therefore, immunohistochemistry for ZEB2 and E-cadherin on serial tissue sections was performed instead. The results showed an inverse correlation between the expression patterns of ZEB2 and E-cadherin: the expression of E-cadherin was detected in those trophoblast cells which were ZEB2-negative but lost or significantly reduced in those trophoblast cells which were ZEB2-positive (Fig. 6).

Previous studies indicated that the transcription factor ZEB2 can suppress E-cadherin expression by binding to the E-box elements in the E-cadherin promoter
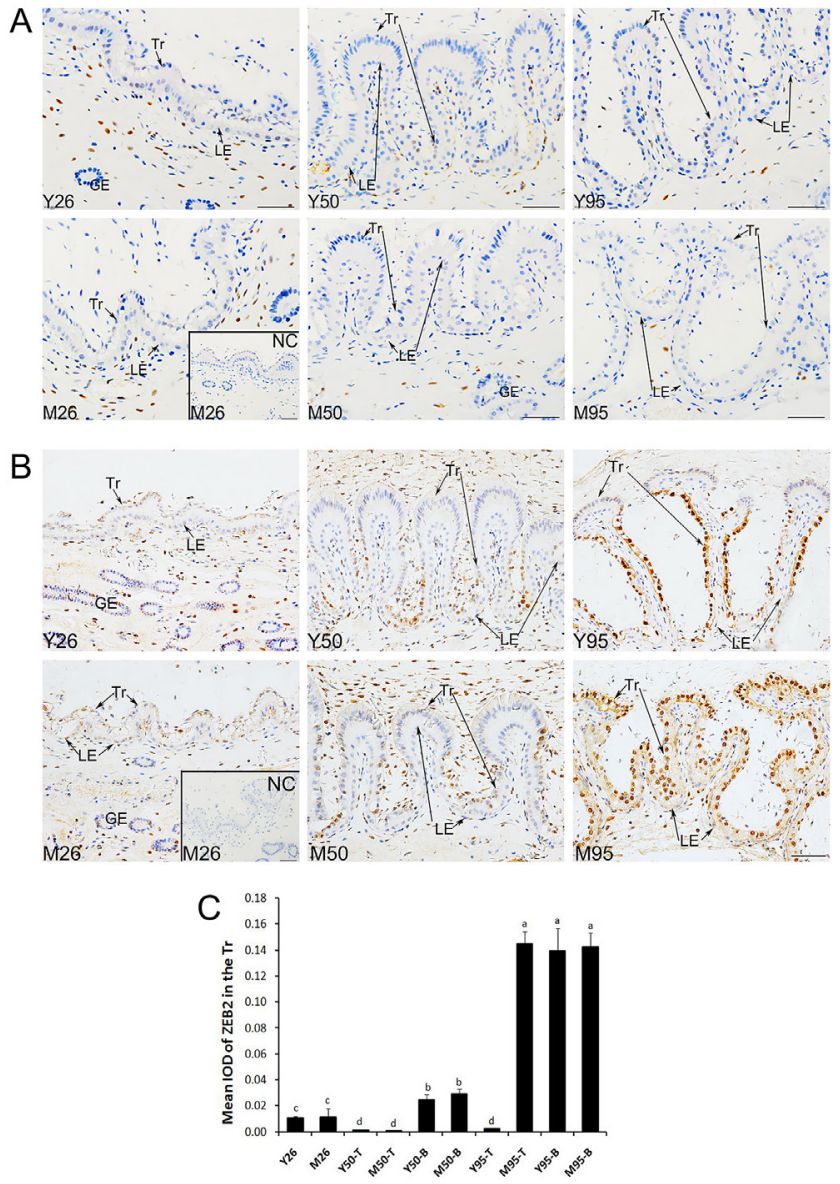

Figure 5 Immunohistochemical analysis of ZEB1 and ZEB2 at the maternal-fetal interface in Yorkshire $(\mathrm{Y})$ and Meishan $(\mathrm{M})$ pigs. (A) ZEB1 expression pattern at the maternal-fetal interface during gestation. Positive signals of ZEB1 were only observed in a few stromal cells (arrows) on days 26, 50 and 95 of gestation in $Y$ and $M$ pigs. (B) ZEB2 expression pattern at the maternal-fetal interface during gestation. On day 26 of gestation, ZEB2 staining were observed in some stromal cells but few positive signals were detected in the trophoblast cells in $\mathrm{Y}$ and $\mathrm{M}$ pigs. On day 50, some positive signals were detected in the trophoblast cells only located at the base and side of the placental folds in the two breeds in $\mathrm{Y}$ and $\mathrm{M}$ pigs. On day 95 of gestation, positive signals were observed in $\operatorname{Tr}$ cells located at the base and side of the placental folds in Y, while in $\mathrm{M}$, positive signals were observed in all Tr cells. All the positive staining of ZEB2 in $\operatorname{Tr}$ cells was located in the nuclei. Tr, trophoblast; LE, endometrial luminal epithelium; GE, glandular epithelium. Insert: NC, negative control; scale bars $=40 \mu \mathrm{m}$. (C) Quantitative analysis of ZEB2 by measuring the average integrated optical density (IOD) in trophoblasts during pregnancy. Means of immunoreaction intensity with different superscript letters differ significantly at $P<0.05$. The data were shown as the mean \pm S.D. T, top of placental folds; $B$, bottom of placental folds.

(Comijn et al. 2001). Therefore, we investigated whether ZEB2 can bind to E-cadherin promoter in porcine trophoblast cells. Due to our findings in this study that ZEB2 was strongly expressed in the nuclei of all trophoblast cells in Meishan placentas on day 95 of gestation (Figs 5 and 6), EMSA were carried out 


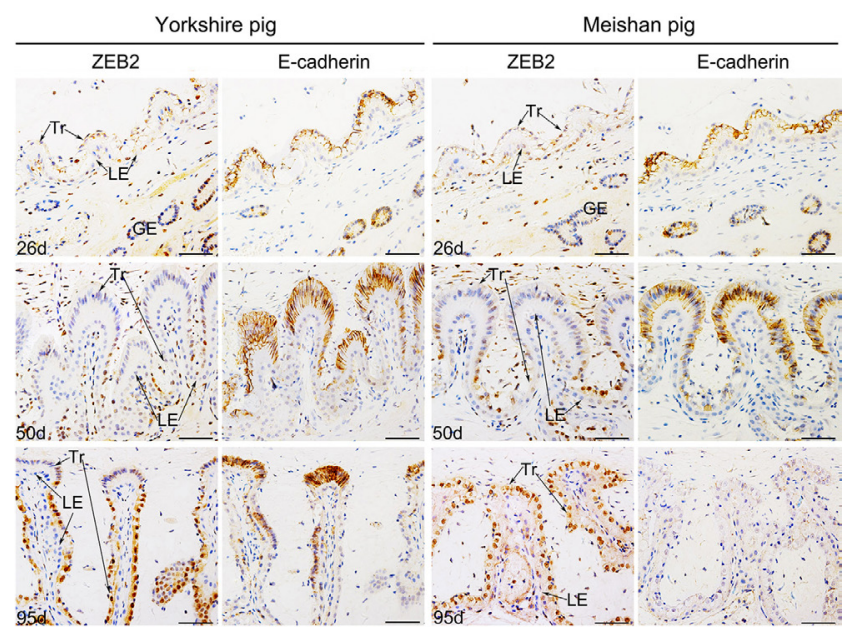

Figure 6 Immunohistochemical analysis of ZEB2 and E-cadherin at the maternal-fetal interface in Yorkshire and Meishan pigs during gestation on serial tissue sections. In trophoblast cells, the distribution patterns of ZEB2 staining were exactly opposite to the distribution patterns of E-cadherin staining during gestation in the two breeds. Tr, trophoblast; LE, endometrial luminal epithelium; GE, glandular epithelium; d, day of gestation; scale bars $=40 \mu \mathrm{m}$.

with nuclear extracts from Meishan placental tissues obtained on day 95 of gestation. A single protein-DNA shift band was present in EMSA, indicating that the oligonucleotide containing E2-box element in porcine E-cadherin promoter bound nuclear proteins (Fig. 7A). Furthermore, the addition of antibody ZEB2 resulted in a supershift band in addition to the protein-DNA shift band (Fig. 7B). These data confirm that the transcription factor ZEB2 in the nuclear protein complex was bound to the E2-box site of the porcine E-cadherin promoter. Taken together, our findings indicate that the expression of E-cadherin in trophoblast cells in porcine placental folds might be regulated by the transcription factor ZEB2.

\section{The expression patterns of EMT marker proteins in trophoblast cells during gestation in Yorkshire and Meishan pigs}

As the loss of E-cadherin in epithelial cell is the most important cause of EMT, to determine whether trophoblast cells underwent EMT during gestation, we investigated the expression patterns of an epithelial marker (cytokeratin) and a mesenchymal marker (vimentin), respectively, in uteroplacental interface sections from days 26, 50 and 95 of gestation in Yorkshire and Meishan pigs. The results showed that in contrast to stromal cells, which were cytokeratinnegative and vimentin-positive, all the trophoblast cells were positive for cytokeratin and negative for vimentin (Fig. 8). These results suggest that all the trophoblast cells did not undergo EMT but still preserved epithelial features during placental development.

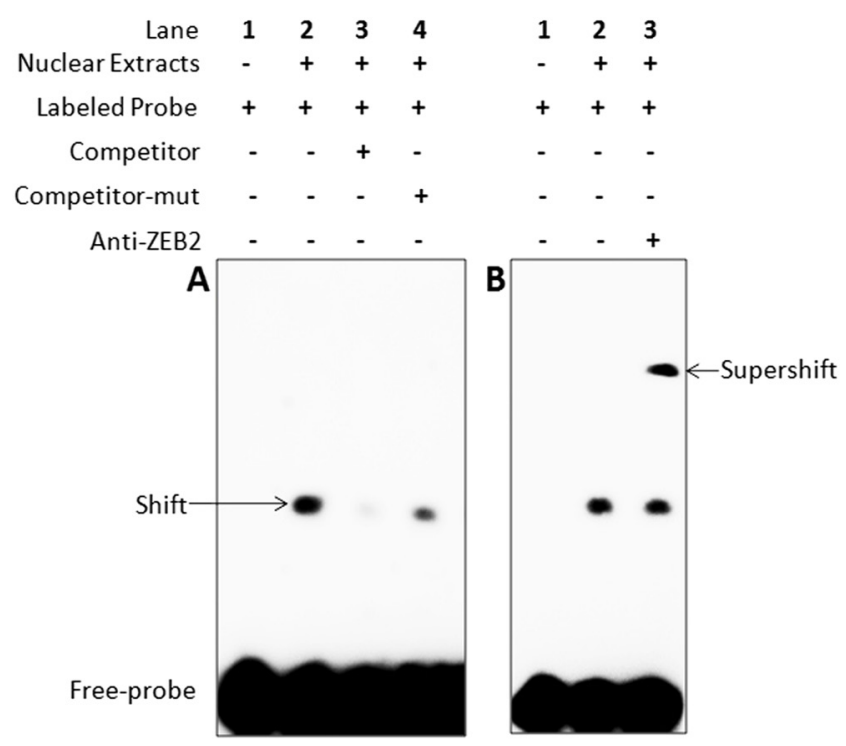

Labeled probe:5' bio-TGCGGCTGGCAGGTGCACCTTCAG3'

Competitor: 5' TGCGGCTGGCAGGTGCACCTTCAG 3'

Mut-Competitor: 5' TGCGGCTGGACTACTCACCTTCAG 3'

ZEB2 Binding Site
(E2-box)

Figure 7 EMSA showing binding of ZEB2 to the porcine E-cadherin promoter. (A) EMSA identifying that the oligonucleotide containing E2-box element in porcine E-cadherin promoter bound nuclear proteins. (B) EMSA identifying that ZEB2 in the nuclear protein complex bound to the E2-box site of the porcine E-cadherin promoter. The supershift indicates the complex of DNA-proteinantibody. The nuclear extracts were from placental tissues of Meishan pigs on day 95 of gestation. The sequences of different probes were shown under the panel.

\section{Discussion}

In this study, we found that all the trophoblast cells were of similar shape in the two pig breeds on day 26 of gestation (the initiation stage of placental folds development (Dantzer \& Leiser 1994, Liu et al. 2015)). However, as the epithelial bilayer developed, two types of trophoblast cells which differed in shape appeared at the top and base of the placental folds: the trophoblast cells located at the top of the placental folds were high columnar, while those cells at the base of the placental folds were cuboidal in shape. These findings are consistent with the previous observations obtained from the western pig breeds (Friess et al. 1980, Vallet et al. 2013). However, in this study, differences in morphology of trophoblast cells between Yorkshire and Meishan pigs were observed during late gestation. In Yorkshire pigs, two different types of trophoblast cell were still present at the top and base of the placental folds on day 95 of gestation, while in Meishan pigs, all trophoblast cells were cuboidal in shape.

Changes in the expression of E-cadherin are associated with alterations of adhesion strength between epithelial cells (Hay 1995, Daikoku et al. 2011). Our results 


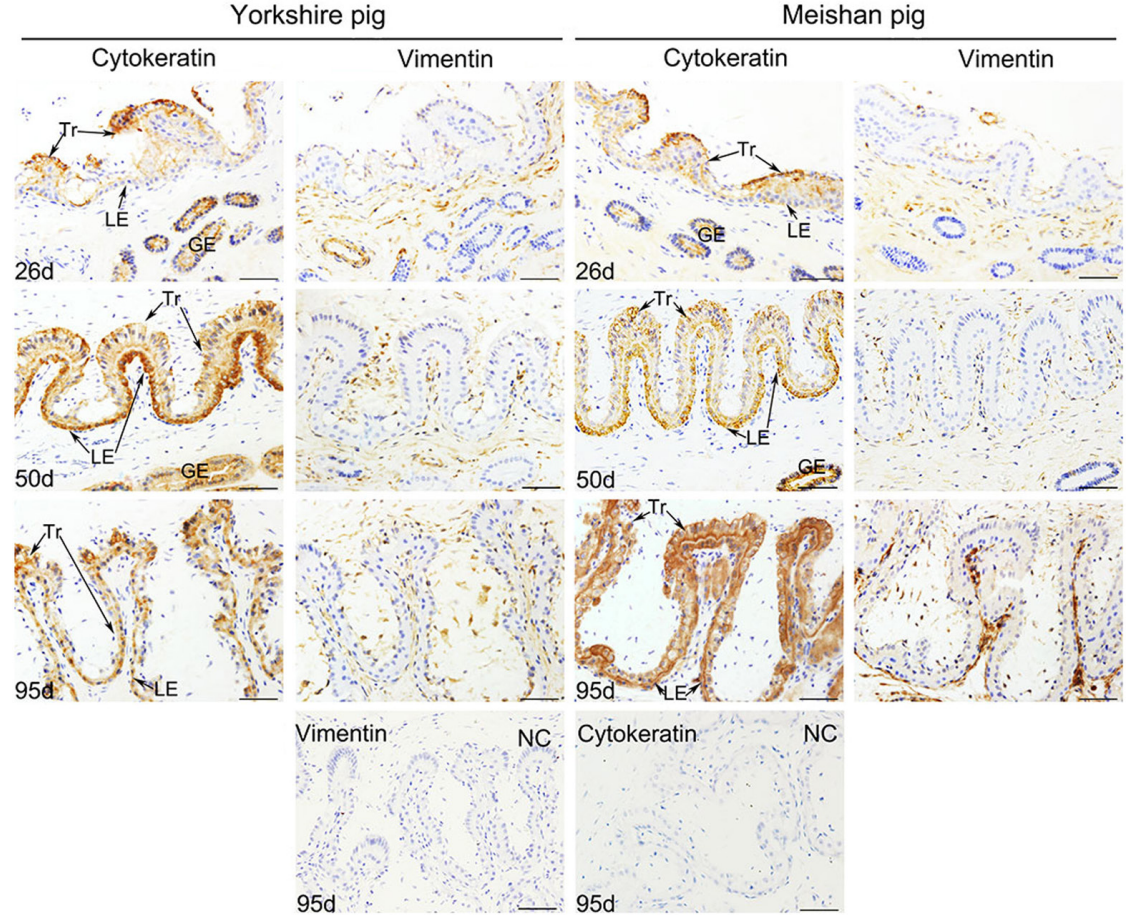

Figure 8 Immunohistochemical analysis of cytokeratin and vimentin at the maternal-fetal interface in Yorkshire and Meishan pigs on days 26, 50 and 95 of gestation on serial tissue sections. Positive signals of cytokeratin were observed in Tr, LE and GE cells, while positive signals of vimentin were observed in stromal cells during gestation in the two breeds. Tr, trophoblast; LE, endometrial luminal epithelium; GE, glandular epithelium; d, day of gestation. Insert: NC, negative control; scale bars $=40 \mu \mathrm{m}$. showed that E-cadherin was strongly expressed by the high columnar trophoblast cells but only weakly expressed by cuboidal trophoblast cells in the placental folds. The adhesive function of E-cadherin in the adherent junction crucially depends on the association with $\beta$-catenin which links the cytoplasmic terminal tail of E-cadherin to the actin cytoskeleton (Auersperg et al. 1999, Leckband \& Sivasankar 2012). Consistent with the expression pattern of E-cadherin, we found that the high columnar trophoblast cells expressed the membranous $\beta$-catenin without detectable nuclear $\beta$-catenin; however, the cuboidal trophoblast cells were negative for both membranous and nuclear $\beta$-catenin expressions. Furthermore, data from our co-IP assays confirmed that E-cadherin interacts with $\beta$-catenin in porcine trophoblast cells, suggesting the formation of E-cadherin- $\beta$-catenin complex in these E-cadherinand $\beta$-catenin-positive trophoblast cells. Therefore, the expression of the E-cadherin- $\beta$-catenin complex is closely associated with the morphological features of trophoblast cells in pigs during gestation, suggesting a role of E-cadherin in regulating the trophoblast cell shape changes as the placental development in pigs.

E-cadherin- $\beta$-catenin complex is necessary for the maintenance of normal intercellular adhesion and epithelial phenotype, and the decreased expression of E-cadherin- $\beta$-catenin complex can lead to increased cell proliferation and invasive abilities (Ciriello et al. 2015, Pereira et al. 2016). Previously, we found that the proliferation marker Ki67 and heparanase, an enzyme which degrades the extracellular matrix and basement membrane, were strongly expressed in the cuboidal trophoblast cells (Miles et al. 2009, Hong et al. 2014, Vallet et al. 2014). Therefore, we propose that markedly downregulated expression of E-cadherin and $\beta$-catenin in the cuboidal trophoblast cells may cause reduced cell-cell adhesion between these cells, resulting in an increase in proliferation of the cuboidal trophoblast cells. On the other hand, the results of PAS staining revealed much more intense staining in the E-cadherin$\beta$-catenin-positive high columnar trophoblast cells than in the cuboidal trophoblast cells. Taken together, our findings support the idea that the cuboidal trophoblast cells, which expressed weak E-cadherin$\beta$-catenin complex, may have a role in promoting the development of the placenta, while the E-cadherin$\beta$-catenin-positive high columnar trophoblast cells may participate in macromolecular transport across the

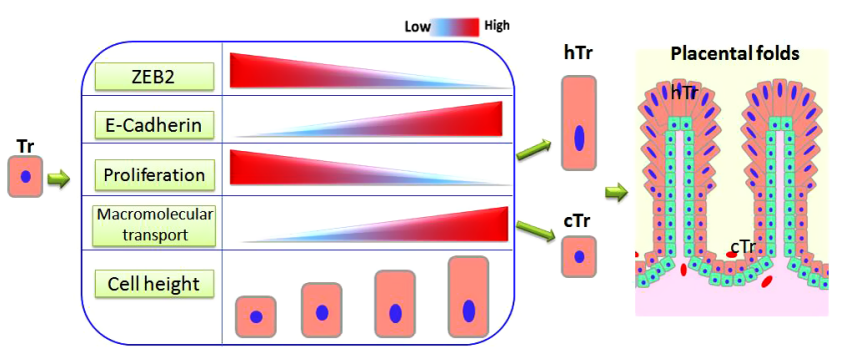

Figure 9 Diagram illustrating the trophoblast cell differentiation mechanisms in pigs. The $\operatorname{Tr}$ (trophoblast cells) differentiate into two different cell types: the $\mathrm{h} \operatorname{Tr}$ (high columnar trophoblast cells) and $\mathrm{c} T \mathrm{r}$ (cuboidal trophoblast cells). ZEB2 can modulate the trophoblast cell differentiation by regulating the E-cadherin expression. 
placenta barrier (Friess et al. 1980, Vallet et al. 2013, 2014, Hong et al. 2014).

On day 95 of gestation, the size of the cuboidal trophoblast cells of the placenta folds in Yorkshire pigs was much smaller than those in Meishan pigs (Table 1). Previous investigations on placental morphology of western pig breeds indicated that the cuboidal trophoblast cells located at the base of the placenta folds mainly contribute to the exchange of the gaseous and nutrients that could be diffusible through the cell membrane (Friess et al. 1980). It has been demonstrated that as the length of the side of a cube decreases, the surface area:volume ratio increases; consequently, the rate of the diffusible substance exchange increases (Syková \& Nicholson 2008). Thus, the cuboidal trophoblast cells with smaller size may be able to take up and eliminate the diffusible substance efficiently during late gestation in Yorkshire pigs compared with Meishan pigs. Combined with the previous observations that the trophoblast cells in Meishan pigs have increased the expression of the nutrient-specific transporters during late gestation (Hong et al. 2016), it seems like the two pig breeds may have their preferential mechanisms to influence the efficiency of nutrient transfer across the placenta barrier during late gestation.

Based on our findings that changes in shape of the trophoblast cells are mediated by E-cadherin- $\beta$-catenin complex, we carried out further investigations to reveal the regulators involved in the $\mathrm{E}$-cadherin expression. We showed that although ZEB1 was not expressed by the two types of trophoblast cells, the expression pattern of ZEB2 was inversely correlated with that of E-cadherin in the two types of trophoblast cell during gestation in the two breeds, as illustrated in our summary (Fig. 9). The results of immunohistochemistry for ZEB2 and E-cadherin on the serial tissue sections confirmed that in contrast to E-cadherin that was expressed by the columnar trophoblast cells, ZEB2 was predominately localized in the nucleus of the cuboidal trophoblast cells but undetectable in the columnar trophoblast cells (Fig. 6). In addition, EMSA results showed that ZEB2 can bind to the E-cadherin promoter in pig placenta. These findings raise the possibility that the expression of E-cadherin in porcine trophoblast cell during placental development may be regulated by the transcription factor ZEB2, but not ZEB1. Loss of the cell surface E-cadherin expression by ZEB2 regulation of epithelial cells is usually associated with a fundamental EMT event (Korpal et al. 2008, Schmalhofer et al. 2009), a process by which epithelial cells lose their cell adhesion capacities and acquire a mesenchymal phenotype with higher migratory and invasive properties (Thiery et al. 2009). However, in this study, the decreased E-cadherin expression did not induce the expression of the mesenchymal marker vimentin; in contrast, the E-cadherin-negative cuboidal trophoblast cells expressed the epithelial marker, cytokeratin. Taken together, our findings suggest that the ZEB2 may only downregulate the expression of cell surface E-cadherin in trophoblast cells but did not cause a full EMT.

We found that the morphological features of trophoblast cells (including cell shape, expression patterns of E-cadherin- $\beta$-catenin complex and ZEB2) in Meishan pigs were different from those in Yorkshire pigs during late gestation. Previous studies indicate that porcine placenta size is influenced by litter size and fetus weight at late gestation (Vallet \& Freking 2007). In this study, the number of fetuses recovered was similar in the Yorkshire gilts but varied greatly in the Meishan gilts from which the samples of uteroplacental interface were collected during late gestation. However, the morphological features of trophoblast cells were the same within these Meishan pigs. In addition, although weight of fetuses recovered was also varied either in the Yorkshire gilts or in the Meishan gilts, no association was observed between the morphological features of trophoblast cells and fetus weight. These suggest that changes in the morphological features of trophoblast cells were independent of the level of uterine crowding and fetus weight during late gestation. Further investigations would be needed to illustrate whether the different morphological features of trophoblast cells in the two breeds could be a cause resulting in the breed difference in placental efficiency.

Collectively, during placental development in pigs, trophoblast cells undergo morphological remodeling to differentiate into two different cell types, high columnar and cuboidal trophoblast cells, respectively. This study provided additional evidence of the specific role of the two cell types in the placental development. In addition, as illustrated in Fig. 9, we conclude that (1) the E-cadherin/ $\beta$-catenin complex plays an important role in mediating the morphological remodeling of porcine trophoblast cells and (2) the transcription factor ZEB2 might participate in the repression of E-cadherin expression but does not induce a full EMT.

\section{Supplementary data}

This is linked to the online version of the paper at http://dx.doi.org/10.1530/REP-17-0254.

\section{Declaration of interest}

The authors declare that there is no conflict of interest that could be perceived as prejudicing the impartiality of the research reported.

\section{Funding}

This work was funded by the Natural Science Foundation of China (31572370), the National Basic Research Program of China (2014CB138500), the National Transgenic Project of China (2016ZX08006003-004) and HZAU pre-research project of China. 


\section{Acknowledgements}

The authors would like to thank Dr Hongmei Wang (Institute of Zoology, Chinese Academy of Sciences) for kindly providing anti-ZEB1 antibody.

\section{References}

Auersperg N, Pan J, Grove BD, Peterson T, Fisher J, Maines-Bandiera S, Somasiri A \& Roskelley CD 1999 E-cadherin induces mesenchymalto-epithelial transition in human ovarian surface epithelium. PNAS 96 6249-6254. (doi:10.1073/pnas.96.11.6249)

Ciriello G, Gatza ML, Beck AH, Wilkerson MD, Rhie SK, Pastore A, Zhang H, McLellan M, Yau C \& Kandoth C 2015 Comprehensive molecular portraits of invasive lobular breast cancer. Cell 163 506-519. (doi:10.1016/j.cell.2015.09.033)

Comijn J, Berx G, Vermassen P, Verschueren K, van Grunsven L, Bruyneel E, Mareel M, Huylebroeck D \& Van Roy F 2001 The twohanded $\mathrm{E}$ box binding zinc finger protein SIP1 downregulates $\mathrm{E}$-cadherin and induces invasion. Molecular Cell 7 1267-1278. (doi:10.1016/ S1097-2765(01)00260-X)

Daikoku T, Cha J, Sun X, Tranguch S, Xie H, Fujita T, Hirota Y, Lydon J, DeMayo F \& Maxson R 2011 Conditional deletion of Msx homeobox genes in the uterus inhibits blastocyst implantation by altering uterine receptivity. Developmental Cell 21 1014-1025. (doi:10.1016/j. devcel.2011.09.010)

Dantzer V 1985 Electron microscopy of the initial stages of placentation in the pig. Anatomy and Embryology 172 281-293. (doi:10.1007/ BF00318976)

Dantzer V \& Leiser R 1994 Initial vascularisation in the pig placenta: I. Demonstration of nonglandular areas by histology and corrosion casts. Anatomical Record 238 177-190. (doi:10.1002/ar.1092380204)

Du L, Kuang L, He F, Tang W, Sun W \& Chen D 2016 Mesenchymal-toepithelial transition in the placental tissues of patients with preeclampsia. Hypertension Research 40 67-72. (doi:10.1038/hr.2016.97)

Eger A, Aigner K, Sonderegger S, Dampier B, Oehler S, Schreiber M, Berx G, Cano A, Beug H \& Foisner R 2005 DeltaEF1 is a transcriptional repressor of $\mathrm{E}$-cadherin and regulates epithelial plasticity in breast cancer cells. Oncogene 24 2375-2385. (doi:10.1038/sj.onc.1208429)

Enders A \& Carter AM 2004 What can comparative studies of placental structure tell us?-A review. Placenta 25 S3-S9. (doi:10.1016/j. placenta.2004.01.011)

Ford S \& Youngs C 1992 Early embryonic development in prolific Meishan pigs. Journal of Reproduction and Fertility Supplement 48 271-278.

Friess AE, Sinowatz F, Skolek-Winnisch R \& Träutner W 1980 The placenta of the pig. Anatomy and Embryology 158 179-191. (doi:10.1007) BF00315905)

Hay E 1995 An overview of epithelio-mesenchymal transformation. Cells Tissues Organs 154 8-20. (doi:10.1159/000147748)

Hong L, Hou C, Li X, Li C \& Yu M 2013 Expression pattern of CD34 at the maternal-foetal interface during pregnancy in pigs. Reproduction in Domestic Animals 48 762-767. (doi:10.1111/rda.12157)

Hong L, Hou C, Li X, Li C, Zhao S \& Yu M 2014 Expression of heparanase is associated with breed-specific morphological characters of placental folded bilayer between Yorkshire and Meishan pigs. Biology of Reproduction 90 56. (doi:10.1095/biolreprod.113.114181)

Hong L, Xu X, Huang J, Lei M, Xu D, Zhao S \& Yu M 2016 Difference in expression patterns of placental cholesterol transporters, ABCA1 and SR-BI, in Meishan and Yorkshire pigs with different placental efficiency. Scientific Reports 6 20503. (doi:10.1038/srep20503)

Kim J \& Vallet J 2007 Placental expression of the membrane form of folate binding protein during pregnancy in swine. Theriogenology $\mathbf{6 7}$ 1279-1284. (doi:10.1016/j.theriogenology.2007.01.016)

Kokkinos M, Murthi P, Wafai R, Thompson E \& Newgreen D 2010 Cadherins in the human placenta-epithelial-mesenchymal transition (EMT) and placental development. Placenta 31 747-755. (doi:10.1016/j. placenta.2010.06.017)

Korpal M, Lee ES, Hu G \& Kang Y 2008 The miR-200 family inhibits epithelial-mesenchymal transition and cancer cell migration by direct targeting of E-cadherin transcriptional repressors ZEB1 and ZEB2.
Journal of Biological Chemistry 283 14910-14914. (doi:10.1074/jbc. C800074200)

Krenacs L, Krenacs T, Stelkovics E \& Raffeld M 2010 Heat-induced antigen retrieval for immunohistochemical reactions in routinely processed paraffin sections. Immunocytochemical Methods and Protocols $\mathbf{5 8 8}$ 103-119. (doi:10.1007/978-1-59745-324-0_14)

Leckband D \& Sivasankar S 2012 Cadherin recognition and adhesion. Current Opinion in Cell Biology 24 620-627. (doi:10.1016/j. ceb.2012.05.014)

Leiser R \& Dantzer V 1988 Structural and functional aspects of porcine placental microvasculature. Anatomy and Embryology 177 409-419. (doi:10.1007/BF00304738)

Leiser R, Pfarrer C, Abd-Elnaeim M \& Dantzer V 1998 Feto-maternal anchorage in epitheliochorial and endotheliochorial placental types studied by histology and microvascular corrosion casts. Placenta 19 21-39. (doi:10.1016/S0143-4004(98)80031-3)

Liu R, Wang M, Su L, Li X, Zhao S \& Yu M 2015 The expression pattern of microRNAs and the associated pathways involved in the development of porcine placental folds that contribute to the expansion of the exchange surface area. Biology of Reproduction 114 126540. (doi:10.1095/ biolreprod.114.126540)

Mesa H, Safranski T, Cammack K, Weaber R \& Lamberson W 2006 Genetic and phenotypic relationships of farrowing and weaning survival to birth and placental weights in pigs. Journal of Animal Science 84 32-40. (doi:10.2527/2006.84132x)

Miles JR, Vallet JL, Freking BA \& Nonneman DJ 2009 Molecular cloning and characterisation of heparanase mRNA in the porcine placenta throughout gestation. Reproduction, Fertility and Development 21 757-772. (doi:10.1071/RD09041)

Pereira CH, Morais MO, Martins AFL, Soares MQS, Alencar RdCG, Batista AC, Leles CR \& Mendonça EF 2016 Expression of adhesion proteins (E-cadherin and $\beta$-catenin) and cell proliferation (Ki-67) at the invasive tumor front in conventional oral squamous cell and basaloid squamous cell carcinomas. Archives of Oral Biology 61 8-15. (doi:10.1016/j.archoralbio.2015.10.003)

Raub TJ, Bazer FW \& Roberts RM 1985 Localization of the iron transport glycoprotein, uteroferrin, in the porcine endometrium and placenta by using immunocolloidal gold. Anatomy and Embryology 171 253-258. (doi:10.1007/BF00341420)

Reardon SN, King ML, MacLean JA, Mann JL, DeMayo FJ, Lydon JP \& Hayashi K 2012 CDH1 is essential for endometrial differentiation, gland development, and adult function in the mouse uterus. Biology of Reproduction 86 141. (doi: 10.1095/biolreprod.112.098871)

Schmalhofer O, Brabletz S \& Brabletz T 2009 E-cadherin, $\beta$-catenin, and ZEB1 in malignant progression of cancer. Cancer and Metastasis Reviews 28 151-166. (doi:10.1007/s10555-008-9179-y)

Song G, Bailey DW, Dunlap KA, Burghardt RC, Spencer TE, Bazer FW \& Johnson GA 2010 Cathepsin B, cathepsin L, and cystatin C in the porcine uterus and placenta: potential roles in endometrial/placental remodeling and in fluid-phase transport of proteins secreted by uterine epithelia across placental areolae. Biology of Reproduction 82 854-864. (doi:10.1095/biolreprod.109.080929)

Syková E \& Nicholson C 2008 Diffusion in brain extracellular space. Physiological Reviews 88 1277-1340. (doi:10.1152/physrev.00027.2007) Takeichi M 1995 Morphogenetic roles of classic cadherins. Current Opinion in Cell Biology 7 619-627. (doi:10.1016/0955-0674(95)80102-2)

Thiery JP 2002 Epithelial-mesenchymal transitions in tumour progression. Nature Reviews Cancer 2 442-454. (doi:10.1038/nrc822)

Thiery JP, Acloque H, Huang RY \& Nieto MA 2009 Epithelial-mesenchymal transitions in development and disease. Cell 139 871-890. (doi:10.1016/ j.cell.2009.11.007)

Tuchscherer M, Puppe B, Tuchscherer A \& Tiemann U 2000 Early identification of neonates at risk: traits of newborn piglets with respect to survival. Theriogenology 54 371-388. (doi:10.1016/S0093$691 \times(00) 00355-1)$

Vallet J \& Freking B 2007 Differences in placental structure during gestation associated with large and small pig fetuses. Journal of Animal Science $\mathbf{8 5}$ 3267-3275. (doi:10.2527/jas.2007-0368)

Vallet JL, Miles JR \& Freking BA 2010 Effect of fetal size on fetal placental hyaluronan and hyaluronoglucosaminidases throughout gestation in the pig. Animal Reproduction Science 118 297-309. (doi:10.1016/j. anireprosci.2009.06.019) 
Vallet J, McNeel A, Johnson G \& Bazer F 2013 Triennial reproduction symposium: limitations in uterine and conceptus physiology that lead to fetal losses. Journal of Animal Science 91 3030-3040. (doi:10.2527/ jas.2012-6138)

Vallet JL, McNeel AK, Miles JR \& Freking BA 2014 Placental accommodations for transport and metabolism during intra-uterine crowding in pigs. Journal of Animal Science and Biotechnology 555. (doi:10.1186/2049-1891-5-55)

Van Aken E, De Wever O, da Rocha CA \& Mareel M 2001 Defective E-cadherin/catenin complexes in human cancer. Virchows Archiv 439 725-751. (doi:10.1007/s004280100516)

Youngs C, Ford S, McGinnis L \& Anderson L 1993 Investigations into the control of litter size in swine: I. Comparative studies on in vitro development of Meishan and Yorkshire preimplantation embryos. Journal of Animal Science 71 1561-1565. (doi:10.2527/1993.7161561x)

Zhang K, Yang G, Wu W, Zhang J, Xia X, Jiang T, Cao J, Huang K, Qiu Z \& Huang C 2016 Decreased expression of caveolin-1 and E-cadherin correlates with the clinicopathologic features of gastric cancer and the EMT process. Recent Patents on Anti-Cancer Drug Discovery 11 236-244. (doi:10.2174/1574892811666160128151437)

Zhou Y, Damsky CH \& Fisher SJ 1997 Preeclampsia is associated with failure of human cytotrophoblasts to mimic a vascular adhesion phenotype. One cause of defective endovascular invasion in this syndrome?. Journal of Clinical Investigation 99 2152. (doi:10.1172/JCl119388)

Received 27 April 2017

First decision 25 May 2017

Revised manuscript received 8 August 2017

Accepted 14 September 2017 